\title{
FREE AREA ESTIMATION IN A DYNAMIC GERM-GRAIN MODEL WITH RENEWAL DROPPING PROCESS
}

\author{
MARCELLO DE GIOSA
}

Received 20 December 2004; Revised 8 April 2005; Accepted 30 April 2005

A partially observed dynamic germ-grain model with renewal dropping process is considered. The expected fraction of free area function is estimated by a product integral-type estimator. Uniform consistency and asymptotic Gaussianity of the estimator are proved. Confidence bands and simulation results are also provided.

Copyright (c) 2006 Marcello De Giosa. This is an open access article distributed under the Creative Commons Attribution License, which permits unrestricted use, distribution, and reproduction in any medium, provided the original work is properly cited.

\section{Introduction}

In this paper we study an estimation problem for the expected fraction of free area function (EFFAF) of a partially observed dynamic germ-grain model with renewal dropping process. The problem is studied in $\mathbb{R}^{2}$, but all results may be extended to $\mathbb{R}^{d}, d \geq 2$.

The mechanism is as follows. At any time of a renewal process, a disk of random bounded area drops on the plane $\mathbb{R}^{2}$ with center uniformly distributed in a convex region $C$. At any time $t$, the union of dropped disks forms a germ-grain model $\Theta(t)$ (see [11]). As a function of $t$, and because of its evolution in time, we call $\Theta=\{\Theta(t): t \geq 0\}$ dynamic germ-grain model (DGGM).

We are interested in the FFAF of a convex closed set $B \subset C$. It is the function $\Delta=\{\Delta(t)$ : $t \geq 0\}$ defined by

$$
\Delta(t):=\frac{\ell(B \backslash \Theta(t))}{\ell(B)}, \quad t \geq 0
$$

where $\ell$ denotes Lebesgue measure and $B \backslash \Theta(t)=B \cap(\Theta(t))^{c}$ is the set difference.

The EFFAF $\omega=\{\omega(t): t \geq 0\}$ is defined by

$$
\omega(t):=\mathbb{E}[\Delta(t)]=\mathbb{E}\left[\frac{\ell(B \backslash \Theta(t))}{\ell(B)}\right], \quad t \geq 0
$$

Hindawi Publishing Corporation Journal of Applied Mathematics and Stochastic Analysis Volume 2006, Article ID 50786, Pages 1-14

DOI 10.1155/JAMSA/2006/50786 
Note that if the realization of the DGGM $\Theta$ is completely observable, the FFAF is (more or less easily) computable. Furthermore, if $\Theta$ is spatially partially observable within a bounded windows, the EFFAF $\omega$ may be estimated by standard methods of spatial statistics (see $[10,11])$.

However, in this paper, having in mind the example below, we consider a different situation. That is, we suppose that the dropping times and the areas of the dropping disks are observable but the positions of the dropped disks are not, so that a different estimation problem arises for $\omega$.

Example 1.1 (bombing problem). Suppose a bombing activity is taking place on a region $C \subset \mathbb{R}^{2}$. Bombs of random destructive power are dropped on $C$ at a time which is related to a renewal process. Each bomb will strike a random point in $C$ and destroy a circular region with its center in the struck point and an area proportional to its destructive power. An observer would like to know the fraction of nondestroyed area function of a target $B \subset$ $C$, that is, the realization of $\Delta$. He is able to register the throwing times and the destructive power of each bomb. Because of the presence of obscuring objects (clouds, hills, etc.), he cannot observe the point struck by each bomb. So an estimate of $\omega$ is required.

We are looking for an estimator of $\omega$ that enjoys good asymptotic properties as the region $C$ grows. Note that when $C$ is big enough, assuming that the disk areas are bounded, the edge effects may be considered negligible.

As is well known in geometric probability,

$$
\omega(t)=\mathbb{P}[0 \notin \Theta(t)]=\mathbb{P}[\tau>t], \quad t \geq 0,
$$

where $0 \in B$ is a fixed test point, and $\tau$ is the hitting time (see [11]) of the point 0 by the DGGM $\Theta$. So $\omega$ is a kind of survival function of the position 0 .

Following standard methods of survival analysis, one could think of estimating $\omega$ by a Kaplan-Meier-type estimator. The point is that the needed data, that is, the hitting times $\tau_{1}, \ldots, \tau_{n}$ of conveniently chosen test points $O_{1}, \ldots, O_{n}$, are not observables in our model. So we have to find another estimation method.

In the paper [6], the dropping process of disks was supposed to be a nonhomogeneous Poisson process, a product integral-type estimator was proposed for $\omega$ and its properties where established by extensive use of martingale theory.

In this paper, we consider a more general case of a renewal dropping process. As we will see, the asymptotic result in part (b) of Theorem 2.1 again will suggest to consider a product integral-type estimator in the renewal case. We will also show that the chosen estimator has good asymptotic properties.

The paper is organized as follows. In Section 2, we describe the model in detail. First we prove a result and then derive from it the EFFAF estimator. Section 3 is devoted to asymptotic properties. Uniform consistency and asymptotic Gaussianity of the estimator are proved. Furthermore, an estimator of the variance function is defined and its uniform consistency is proved. In Section 4, the results of Section 3 are used to find confidence bands. Furthermore, in the same section, we provide results of numerical simulations. In Section 5, we briefly consider the Poisson dropping process case. 
We assume all random variables considered in the paper are defined on the same probability space $(\Omega, \mathscr{F}, P)$.

In what follows, the symbol $\Rightarrow$ will denote the weak convergence of sequences of random processes or random variables, see [2].

\section{Model, notations, and preliminary results}

As already mentioned, we are looking for an estimator of the EFFAF with good asymptotic properties as the region of interest increases. So we fix the time interval for observations to be, say, $[0, T]$ and, as usual in spatial statistics, consider a convex averaging sequence $\left\{C_{n}: n \geq 1\right\}$, as defined in [5, page 332], that is,

(1) $C_{n} \subset \mathbb{R}^{2}$ is a convex Borel set;

(2) $C_{n} \subset C_{n+1}$, for $n=1,2, \ldots$;

(3) $r\left(C_{n}\right) \rightarrow \infty, n \rightarrow \infty$, where $r\left(C_{n}\right):=\sup \left\{r>0: C_{n}\right.$ contains a ball of radius $\left.r\right\}$.

Note that $c_{n}:=\ell\left(C_{n}\right) \rightarrow \infty$, as $n \rightarrow \infty$.

We have in mind a model in which the mean interdropping time is inversely proportional to the area of the region $C_{n}$. That is, on a bigger region there is a bigger dropping rate. First we consider an underlying renewal process $N=\{N(t): t \geq 0\}$ :

$$
N(t):=\max \left[k: \sum_{1 \leq i \leq k} U_{i} \leq t\right], \quad t \geq 0,
$$

where as usual $U_{i}^{\prime} s$ are i.i.d. positive random variables with mean $\mu_{U}$ and variance $\sigma_{U}^{2}$. If $t<U_{1}$, it is assumed $N(t):=0$. In our model, the dropping process on $C_{n}$ is the renewal process $N_{n}=\left\{N_{n}(t): t \geq 0\right\}$ defined by

$$
N_{n}(t):=N\left(c_{n} t\right), \quad t \geq 0
$$

Hence,

$$
N_{n}(t):=\max \left[k: \sum_{1 \leq i \leq k} U_{n i} \leq t\right], \quad t \geq 0 \text {, where } U_{n i}:=\frac{U_{i}}{c_{n}}
$$

At any renewal time $T_{n i}=U_{n 1}+\cdots+U_{n i}$ of $N_{n}$ a disk $D_{n i}=B\left(X_{n i}, \sqrt{A_{n i} / \pi}\right)$ of random area $A_{n i}$ drops on $\mathbb{R}^{2}$ with random center $X_{n i} \in C_{n}$.

About disk centers and areas, we assume that, for any $n \geq 1$,

(A1) $X_{n 1}, \ldots, X_{n m}, \ldots$ is a sequence of i.i.d. random variables uniformly distributed on $C_{n}$

(A2) $A_{n 1}, \ldots, A_{n m}, \ldots$ is a sequence of i.i.d. bounded random variables with mean $\mu_{A}$, second moment $\mu_{A}^{(2)}$, finite variance $\sigma_{A}^{2}$, and finite first four moments,

(A3) we further assume that the families of random variables $\left\{X_{n m}: m \geq 1\right\},\left\{A_{n m}\right.$ : $m \geq 1\}$ and $\left\{N_{n}(t): t \geq 0\right\}$ are independent of each other.

For any $t \geq 0$, we denote by $\Theta_{n}(t)=\left\{\Theta_{n}(t, \omega), \omega \in \Omega\right\}$ the random closed set (germgrain model) composed by the union of the random disks dropped up to time $t$,

$$
\Theta_{n}(t):=\bigcup_{1 \leq i \leq N_{n}(t)} D_{n i}=\bigcup_{T_{n i} \leq t} D_{n i}, \quad t \geq 0 .
$$


In the following, $\theta_{n}(t), \Delta_{n}(t)$, and $\omega_{n}(t)$ will denote, respectively, the area of $\Theta_{n}(t)$, the fraction area FFAF, and the expected fraction area EFFAF at time $t$. That is,

$$
\theta_{n}(t):=\ell\left(\Theta_{n}(t)\right), \quad \Delta_{n}(t):=1-\frac{\theta_{n}(t)}{c_{n}}, \quad \omega_{n}(t):=\mathbb{E}\left[\Delta_{n}(t)\right] .
$$

Theorem 2.1 below gives an insight into a possible approach in estimating $\omega_{n}(t)$. However, we first recall two useful results about renewal processes (see [2, equation (14.29), page 154] and [8, Theorem 4.2, page 188]). If $N$ is a renewal process, as defined in (2.1), then

$$
\begin{gathered}
\sup _{0 \leq s \leq t} \frac{1}{t}\left|N(s)-\frac{s}{\mu_{U}}\right| \Longrightarrow 0, \quad \text { as } t \longrightarrow \infty, \\
\frac{\mathbb{E}[N(t)]}{t} \longrightarrow \frac{1}{\mu_{U}}, \quad \text { as } t \longrightarrow \infty .
\end{gathered}
$$

These results have the following implications on the dropping processes $\left(N_{n}\right)$. Being

$$
\sup _{0 \leq t \leq T}\left|\frac{N_{n}(t)}{c_{n}}-\frac{t}{\mu_{U}}\right| \leq T \cdot \sup _{0 \leq u \leq c_{n} T} \frac{1}{c_{n} T}\left|N(u)-\frac{u}{\mu_{U}}\right|,
$$

by (2.6) it follows that

$$
\sup _{0 \leq t \leq T}\left|\frac{N_{n}(t)}{c_{n}}-\frac{t}{\mu_{U}}\right| \Longrightarrow 0, \quad \text { as } n \longrightarrow \infty .
$$

By (2.7) it follows that, for any $t \in[0, T]$,

$$
\frac{\mathbb{E}\left[N_{n}(t)\right]}{c_{n}}=t \cdot \frac{\mathbb{E}\left[N\left(c_{n} t\right)\right]}{c_{n} t} \longrightarrow \frac{t}{\mu_{U}}, \quad \text { as } n \longrightarrow \infty
$$

Let us now state and prove the following useful result.

Theorem 2.1. Define, for any $n \geq 1$ and $t \geq 0$,

$$
\mathscr{S}_{n}(t):=\left(1-\frac{\mu_{A}}{c_{n}}\right)^{N_{n}(t)}, \quad \mathscr{S}(t):=\exp \left(-\frac{\mu_{A}}{\mu_{U}} t\right) .
$$

Then the following hold:

(a) $\omega_{n}(t)=\mathbb{E}\left[\mathscr{Y}_{n}(t)\right]$, for any $t \geq 0$;

(b) $\sup _{0 \leq t \leq T}\left|\omega_{n}(t)-\mathscr{Y}(t)\right| \rightarrow 0$, as $n \rightarrow \infty$.

Proof. (a) Let $0 \in B$ be a fixed test point and $D_{n i}$ the generic disk dropped on $C_{n}$. Because of the independence assumption (A3) and uniformity of the disk center distributions, by well-known results in geometric probability, we have

$$
\mathbb{P}\left[0 \notin D_{n i}\right]=1-\frac{\mu_{A}}{c_{n}} .
$$


Again because of the independence assumption,

$$
\begin{aligned}
\omega_{n}(t) & =\mathbb{E}\left[\mathbb{E}\left[\Delta_{n}(t) \mid N_{n}(t)\right]\right]=\mathbb{E}\left[\mathbb{P}\left[0 \notin \Theta_{n}(t) \mid N_{n}(t)\right]\right] \\
& =\mathbb{E}\left[\left(1-\frac{\mu_{A}}{c_{n}}\right)^{N_{n}(t)}\right]=\mathbb{E}\left[\mathscr{Y}_{n}(t)\right],
\end{aligned}
$$

and hence $(a)$ is proved.

(b) Since, for any $t \geq 0$,

$$
\mathscr{Y}_{n}(t):=\exp \left(\frac{N_{n}(t)}{c_{n}} \cdot \frac{\ln \left(1-\mu_{A} / c_{n}\right)}{\mu_{A} / c_{n}} \cdot \mu_{A}\right)
$$

in view of (2.9) and equicontinuity of the exponential in $[-T, 0]$, we have

$$
\sup _{0 \leq t \leq T}\left|\mathscr{S}_{n}(t)-\mathscr{S}(t)\right| \Longrightarrow 0, \quad \text { as } n \longrightarrow \infty
$$

Because $\sup _{0 \leq t \leq T}\left|\mathscr{S}_{n}(t)-\mathscr{Y}(t)\right| \leq 1$ and

$$
\sup _{0 \leq t \leq T}\left|\omega_{n}(t)-\mathscr{S}(t)\right| \leq \mathbb{E}\left[\sup _{0 \leq t \leq T}\left|\mathscr{T}_{n}(t)-\mathscr{S}(t)\right|\right],
$$

then (b) follows by dominated convergence arguments.

We now deduce from Theorem 2.1 a possible approach for estimating the expected fraction area EFFAF $\omega_{n}$. Note that

$$
\mathscr{S}(t)=e^{-\Lambda(t)}=\prod_{s \leq t}(1-d \Lambda(s)), \quad 0 \leq t \leq T,
$$

where $\prod$ denotes product integral (see [1, Section II.6] or [7]), and

$$
\Lambda(s)=\frac{\mu_{A}}{\mu_{U}} s .
$$

Statement (b) in Theorem 2.1 suggests an estimator for $\omega_{n}(t)$ of the following type:

$$
\hat{\mathscr{S}}_{n}(t):=\prod_{s \leq t}\left(1-d \hat{\Lambda}_{n}(s)\right), \quad 0 \leq t \leq T
$$

where $\hat{\Lambda}_{n}$ should be a good estimator for $\Lambda$. A natural estimator for $\Lambda$ is the normalized cumulative sum process $\hat{\Lambda}_{n}=\left\{\hat{\Lambda}_{n}(t) ; 0 \leq t \leq T\right\}$ defined by

$$
\widehat{\Lambda}_{n}(t):=\frac{1}{c_{n}} \sum_{i=1}^{N_{n}(t)} A_{n i}, \quad 0 \leq t \leq T .
$$

Note that, with $\hat{\Lambda}_{n}$ as above, it is true that

$$
\hat{\mathscr{S}}_{n}(t)=\prod_{T_{n i} \leq t}\left(1-\frac{A_{n i}}{c_{n}}\right), \quad 0 \leq t \leq T .
$$




\section{Asymptotic results}

In this section, we state and prove the uniform consistency and asymptotic Gaussianity of the estimator $\hat{\mathscr{S}}_{n}$.

Theorem 3.1 (uniform consistency). With the same definitions and notations as in the previous sections, the process $\hat{\mathscr{Y}}_{n}$ is a uniform consistent estimator of the EFFAF $\omega_{n}$, that is,

$$
\sup _{0 \leq t \leq T}\left|\hat{\mathscr{S}}_{n}(t)-\omega_{n}(t)\right| \Longrightarrow 0, \quad \text { as } n \longrightarrow \infty \text {. }
$$

Proof. Note that

$$
\begin{aligned}
& \sup _{0 \leq t \leq T}\left|\hat{\mathscr{Y}}_{n}(t)-\omega_{n}(t)\right| \\
& \quad \leq \sup _{0 \leq t \leq T}\left|\hat{\mathscr{S}}_{n}(t)-\mathscr{Y}_{n}(t)\right|+\sup _{0 \leq t \leq T}\left|\mathscr{S}_{n}(t)-\mathscr{Y}(t)\right|+\sup _{0 \leq t \leq T}\left|\omega_{n}(t)-\mathscr{Y}(t)\right| .
\end{aligned}
$$

Because of statement (b) in Theorem 2.1 and (2.15), we have only to prove that

$$
\sup _{0 \leq t \leq T}\left|\hat{\mathscr{S}}_{n}(t)-\mathscr{Y}_{n}(t)\right| \Longrightarrow 0, \quad \text { as } n \longrightarrow \infty \text {. }
$$

Note that

$$
\mathscr{S}_{n}(t):=\prod_{s \leq t}\left(1-d \Lambda_{n}(s)\right), \quad \text { with } \Lambda_{n}(s):=\frac{\mu_{A}}{c_{n}} N_{n}(s)
$$

In view of (2.19) and the continuity of the product-integrals (see [1, page 114] or [7]), the convergence in (3.3) will follow if we prove that

$$
\sup _{0 \leq t \leq T}\left|\hat{\Lambda}_{n}(t)-\Lambda_{n}(t)\right| \Longrightarrow 0, \quad \text { as } n \longrightarrow \infty .
$$

For any $\varepsilon>0$ and $m \geq 1$, by (A3),

$$
\begin{aligned}
\mathbb{E}\left[I\left(\sup _{0 \leq t \leq T}\left|\hat{\Lambda}_{n}(t)-\Lambda_{n}(t)\right| \geq \varepsilon\right)-\frac{N_{n}(T) \sigma_{A}^{2}}{c_{n}^{2} \varepsilon^{2}} \mid N_{n}(T)=m\right] \\
=\mathbb{E}\left[I\left(\sup _{k \leq m}\left|\sum_{1 \leq i \leq k}\left(A_{n i}-\mu_{A}\right)\right| \geq c_{n} \varepsilon\right)-\frac{\operatorname{Var}\left(\sum_{1 \leq k \leq m}\left(A_{n i}-\mu_{A}\right)\right)}{c_{n}^{2} \varepsilon^{2}}\right] \\
=\mathbb{P}\left[\sup _{k \leq m}\left|\sum_{1 \leq i \leq k}\left(A_{n i}-\mu_{A}\right)\right| \geq c_{n} \varepsilon\right]-\frac{\operatorname{Var}\left(\sum_{1 \leq k \leq m}\left(A_{n i}-\mu_{A}\right)\right)}{c_{n}^{2} \varepsilon^{2}}
\end{aligned}
$$

and the last term is $\leq 0$ by Kolmogorov inequality. It follows that

$$
\mathbb{E}\left[I\left(\sup _{0 \leq t \leq T}\left|\hat{\Lambda}_{n}(t)-\Lambda_{n}(t)\right| \geq \varepsilon\right)-\frac{N_{n}(T) \sigma_{A}^{2}}{c_{n}^{2} \varepsilon^{2}} \mid N_{n}(T)\right] \leq 0
$$


and then

$$
\begin{aligned}
& \mathbb{E}\left[I\left(\sup _{0 \leq t \leq T}\left|\hat{\Lambda}_{n}(t)-\Lambda_{n}(t)\right| \geq \varepsilon\right)-\frac{N_{n}(T) \sigma_{A}^{2}}{c_{n}^{2} \varepsilon^{2}}\right] \\
& \quad=\mathbb{E}\left[\mathbb{E}\left[I\left(\sup _{0 \leq t \leq T}\left|\hat{\Lambda}_{n}(t)-\Lambda_{n}(t)\right| \geq \varepsilon\right)-\frac{N_{n}(T) \sigma_{A}^{2}}{c_{n}^{2} \varepsilon^{2}} \mid N_{n}(T)\right]\right] \leq 0 .
\end{aligned}
$$

So

$$
\mathbb{P}\left[\sup _{0 \leq t \leq T}\left|\widehat{\Lambda}_{n}(t)-\Lambda_{n}(t)\right| \geq \varepsilon\right] \leq \frac{\mathbb{E}\left[N_{n}(T)\right] \sigma_{A}^{2}}{c_{n}^{2} \varepsilon^{2}}
$$

and (3.5) follows from (2.10).

Theorem 3.2 (asymptotic Gaussianity). The process $\mathcal{M}_{n}^{\mathscr{P}}=\left\{\mathcal{M}_{n}^{\mathscr{S}}(t): 0 \leq t \leq T\right\}$, defined by

$$
M_{n}^{\mathscr{Y}}(t):=\sqrt{c_{n}}\left(\frac{\hat{\mathscr{S}}_{n}(t)-\mathscr{Y}_{n}(t)}{\mathscr{S}_{n}(t)}\right), \quad 0 \leq t \leq T,
$$

converges to $W(v)$ :

$$
M_{n}^{\varphi} \Longrightarrow W(v), \quad \text { as } n \longrightarrow \infty,
$$

where $W$ is a standard Brownian motion on $[0, T]$, and $v=\left\{v(t):=\sigma_{A}^{2} \cdot \phi(t)\right.$, with $\phi(t):=$ $\left.t / \mu_{U}, 0 \leq t \leq T\right\}$.

Proof. Let us define the process $\mathcal{M}_{n}^{\Lambda}=\left\{\mathcal{M}_{n}^{\Lambda}(t): 0 \leq t \leq T\right\}$ by

$$
M_{n}^{\Lambda}(t):=\sqrt{c_{n}}\left(\widehat{\Lambda}_{n}(t)-\Lambda_{n}(t)\right)=\frac{1}{\sqrt{c_{n}}} \sum_{i=1}^{N_{n}(t)}\left(A_{n i}-\mu_{A}\right), \quad 0 \leq t \leq T .
$$

We first prove that

$$
M_{n}^{\Lambda} \Longrightarrow W(v), \quad \text { as } n \longrightarrow \infty \text {. }
$$

The proof of (3.13) will follow the same lines as the proof of [2, Theorem 14.4].

First suppose $T / \mu_{U}<1$. Let us define, for any $t \in[0, T]$,

$$
\Phi_{n}(t):= \begin{cases}\frac{N_{n}(t)}{c_{n}} & \text { if } \frac{N_{n}(t)}{c_{n}} \leq 1, \\ \phi(t) & \text { otherwise. }\end{cases}
$$

Note that, because of (2.9),

$$
\sup _{0 \leq t \leq T}\left|\Phi_{n}(t)-\phi(t)\right| \leq \sup _{0 \leq t \leq T}\left|\frac{N_{n}(t)}{c_{n}}-\frac{t}{\mu_{U}}\right| \Longrightarrow 0, \quad \text { as } n \longrightarrow \infty,
$$


so that

$$
\Phi_{n} \Longrightarrow \phi, \quad \text { as } n \longrightarrow \infty
$$

By Donsker theorem (see [2, Theorem 14.1]), the process $X_{c_{n}}=\left\{X_{c_{n}}(t): 0 \leq t \leq T\right\}$ defined by

$$
X_{c_{n}}(t):=\frac{1}{\sqrt{c_{n}}} \sum_{i=1}^{\left\lfloor c_{n} t\right\rfloor}\left(A_{n i}-\mu_{A}\right), \quad 0 \leq t \leq T
$$

converges to $\sigma_{A} \cdot W$ :

$$
X_{c_{n}} \Longrightarrow \sigma_{A} \cdot W, \quad \text { as } n \longrightarrow \infty \text {. }
$$

By (3.16) and (3.18) it follows (see [2, Theorem 3.9]) that

$$
\left(X_{c_{n}}, \Phi_{n}\right) \Longrightarrow\left(\sigma_{A} \cdot W, \phi\right), \quad \text { as } n \longrightarrow \infty
$$

and, since $W$ is a.s. continuous (see [2, Lemma, page 151]), then

$$
\left(X_{c_{n}} \circ \Phi_{n}\right) \Rightarrow \sigma_{A} \cdot(W \circ \phi), \quad \text { as } n \longrightarrow \infty
$$

Fix now $\varepsilon>0$ and put $\delta:=1-T / \mu_{U}$. We have

$$
\begin{gathered}
\mathbb{P}\left[\sup _{0 \leq t \leq T}\left|M_{n}^{\Lambda}(t)-\left(X_{c_{n}} \circ \Phi_{n}\right)(t)\right|>\varepsilon\right] \leq \mathbb{P}\left[\sup _{0 \leq t \leq T} \frac{N_{n}(t)}{c_{n}}>1\right] \\
\leq \mathbb{P}\left[\sup _{0 \leq t \leq T}\left|\frac{N_{n}(t)}{c_{n}}-\frac{t}{\mu_{U}}\right|>\delta\right],
\end{gathered}
$$

and, because of (2.9), the last probability goes to 0 , as $n \rightarrow \infty$. So

$$
M_{n}^{\Lambda} \Longrightarrow \sigma_{A} \cdot(W \circ \phi), \quad \text { as } n \longrightarrow \infty
$$

and hence (3.13) follows because $\sigma_{A} \cdot(W \circ \phi)$ and $W(v)$ have the same distribution.

If $T / \mu_{U} \geq 1$ and $a>0$ is such that $T / a \mu_{U}<1$, the proof of (3.13) can be arranged as before, by substituting $c_{n}$ with $a c_{n}$.

Now, after having established (3.13), we note that, by Duhamel equation (see [1, equation (2.6.5)]),

$$
M_{n}^{\mathscr{P}}(t):=-\int_{0}^{t} \frac{\hat{\mathscr{S}}_{n}\left(s^{-}\right)}{\mathscr{S}_{n}(s)} d\left(\sqrt{c_{n}}\left(\hat{\Lambda}_{n}(s)-\Lambda_{n}(s)\right)\right)=-\int_{0}^{t} \frac{\hat{\mathscr{S}}_{n}\left(s^{-}\right)}{\mathscr{S}_{n}(s)} d M_{n}^{\Lambda}(s),
$$


so that

$$
\begin{aligned}
\sup _{0 \leq t \leq T}\left|\mathcal{M}_{n}^{\mathscr{S}}(t)-\left(-\mathcal{M}_{n}^{\Lambda}(t)\right)\right| & =\sup _{0 \leq t \leq T}\left|\int_{0}^{t}\left(\frac{\hat{\mathscr{S}}_{n}\left(s^{-}\right)}{\mathscr{S}_{n}(s)}-1\right) d M_{n}^{\Lambda}(s)\right| \\
& \leq \sup _{0 \leq t \leq T}\left|\frac{\hat{\mathscr{S}}_{n}\left(t^{-}\right)}{\mathscr{S}_{n}(t)}-1\right| \cdot \sup _{0 \leq t \leq T} \mathcal{M}_{n}^{\Lambda}(t) .
\end{aligned}
$$

Moreover,

$$
\sup _{0 \leq t \leq T}\left|\frac{\hat{\mathscr{S}}_{n}\left(t^{-}\right)}{\mathscr{S}_{n}(t)}-1\right| \leq \frac{1}{\mathscr{S}_{n}(T)} \cdot \sup _{0 \leq t \leq T}\left|\hat{\mathscr{S}}_{n}\left(t^{-}\right)-\mathscr{Y}_{n}(t)\right|,
$$

and because of (3.3), we have

$$
\sup _{0 \leq t \leq T}\left|\frac{\hat{\mathscr{S}}_{n}\left(t^{-}\right)}{\mathscr{S}_{n}(t)}-1\right| \Longrightarrow 0, \quad \text { as } n \longrightarrow \infty .
$$

Furthermore, (3.13) implies that

$$
\sup _{0 \leq t \leq T} M_{n}^{\Lambda}(t) \Longrightarrow \sup _{0 \leq t \leq T} W(v(t)), \quad \text { as } n \longrightarrow \infty .
$$

Hence,

$$
\sup _{0 \leq t \leq T}\left|M_{n}^{\mathscr{S}}(t)-\left(-M_{n}^{\Lambda}(t)\right)\right| \Longrightarrow 0, \quad \text { as } n \longrightarrow \infty,
$$

and, again because of (3.13), we arrive at the desired convergence (3.11).

The next step is to use Theorems 3.1 and 3.2 and derive confidence bands (strips). First we have to find a good estimator for the variance function $v$. Let us define the process $\hat{v}_{n}=\left\{\hat{v}_{n}(t): 0 \leq t \leq T\right\}$ by

$$
\widehat{v}_{n}(t):=\frac{1}{c_{n}} \sum_{i=1}^{N_{n}(t)}\left(A_{n i}-\frac{1}{N_{n}(t)} \sum_{i=1}^{N_{n}(t)} A_{n i}\right)^{2}, \quad 0 \leq t \leq T .
$$

THeOREm 3.3. The process $\hat{v}_{n}$ is a uniformly consistent estimator of the variance function $v$, that is,

$$
\sup _{0 \leq t \leq T}\left|\hat{v}_{n}(t)-v(t)\right| \Longrightarrow 0, \quad \text { as } n \longrightarrow \infty .
$$

Proof. Note that, for any $t \in[0, T]$, we have

$$
\hat{v}_{n}(t):=\frac{1}{c_{n}} \sum_{i=1}^{N_{n}(t)}\left(A_{n i}-\mu_{A}\right)^{2}-\frac{1}{N_{n}(t)}\left(M_{n}^{\Lambda}(t)\right)^{2} .
$$

So

$$
\left|\hat{v}_{n}(t)-v(t)\right| \leq \mathscr{P}_{n}(t)+\sigma_{A}^{2}\left|\frac{N_{n}(t)}{c_{n}}-\frac{t}{\mu_{U}}\right|+\frac{1}{N_{n}(t)}\left(M_{n}^{\Lambda}(t)\right)^{2}
$$


where, for notational convenience, we have put

$$
\mathscr{B}_{n}(t):=\frac{1}{c_{n}}\left|\sum_{i=1}^{N_{n}(t)}\left(\left(A_{n i}-\mu_{A}\right)^{2}-\sigma_{A}^{2}\right)\right|
$$

The last term in (3.32) goes uniformly to 0 in probability because of (2.9) and (3.13). The second term goes uniformly to 0 in probability because of (2.9). It follows that we have only to show that

$$
\sup _{0 \leq t \leq T} \mathscr{B}_{n}(t) \Longrightarrow 0, \quad \text { as } n \longrightarrow \infty \text {. }
$$

Let $\varepsilon>0$. For any $m \geq 1$, by (A3),

$$
\begin{aligned}
\mathbb{E}\left[I\left(\sup _{0 \leq t \leq T} \mathscr{B}_{n}(t) \geq \varepsilon\right)-\frac{N_{n}(T) \operatorname{Var}\left(\left(A_{n i}-\mu_{A}\right)^{2}\right)}{\mathcal{E}^{2} c_{n}^{2}} \mid N_{n}(T)=m\right] \\
\quad=\mathbb{E}\left[I\left(\sup _{k \leq m}\left|\sum_{1 \leq i \leq k}\left(\left(A_{n i}-\mu_{A}\right)^{2}-\sigma_{A}^{2}\right)\right| \geq \varepsilon c_{n}\right)-\frac{m \operatorname{Var}\left(\left(A_{n i}-\mu_{A}\right)^{2}\right)}{\mathcal{E}^{2} c_{n}^{2}}\right] \\
=\mathbb{P}\left[\sup _{k \leq m}\left|\sum_{1 \leq i \leq k}\left(\left(A_{n i}-\mu_{A}\right)^{2}-\sigma_{A}^{2}\right)\right| \geq \varepsilon c_{n}\right]-\frac{\operatorname{Var}\left(\sum_{1 \leq i \leq m}\left(A_{n i}-\mu_{A}\right)^{2}\right)}{\mathcal{E}^{2} c_{n}^{2}},
\end{aligned}
$$

and the last term is $\leq 0$ by Kolmogorov inequality. It follows that

$$
\mathbb{E}\left[I\left(\sup _{0 \leq t \leq T} \mathscr{B}_{n}(t) \geq \varepsilon\right)-\frac{N_{n}(T) \operatorname{Var}\left(\left(A_{n i}-\mu_{A}\right)^{2}\right)}{\varepsilon^{2} c_{n}^{2}} \mid N_{n}(T)\right] \leq 0,
$$

so that

$$
\begin{array}{r}
\mathbb{E}\left[I\left(\sup _{0 \leq t \leq T} \mathscr{B}_{n}(t) \geq \varepsilon\right)-\frac{N_{n}(T) \operatorname{Var}\left(\left(A_{n i}-\mu_{A}\right)^{2}\right)}{\mathcal{E}^{2} c_{n}^{2}}\right] \\
\quad=\mathbb{E}\left[\mathbb{E}\left[I\left(\sup _{0 \leq t \leq T} \mathscr{B}_{n}(t) \geq \varepsilon\right)-\frac{N_{n}(T) \operatorname{Var}\left(\left(A_{n i}-\mu_{A}\right)^{2}\right)}{\varepsilon^{2} c_{n}^{2}} \mid N_{n}(T)\right]\right] \leq 0 .
\end{array}
$$

Hence,

$$
\mathbb{P}\left[\sup _{0 \leq t \leq T} \mathscr{B}_{n}(t) \geq \varepsilon\right] \leq \frac{\mathbb{E}\left[N_{n}(T)\right]}{\varepsilon^{2} c_{n}^{2}} \operatorname{Var}\left[\left(A_{n i}-\mu_{A}\right)^{2}\right]
$$

and the conclusion follows from (2.10). 


\section{Confidence bands and simulations}

In order to find confidence bands, let us show that the following theorem holds.

THEOREM 4.1. Under the same notations and assumptions as in the previous sections, the following convergence result holds:

$$
\sup _{0 \leq t \leq T}\left|\frac{\sqrt{c_{n}}}{1+\hat{v}_{n}(t)} \frac{\hat{\mathscr{S}}_{n}(t)-\mathscr{S}_{n}(t)}{\hat{\mathscr{S}}_{n}(t)}\right| \Longrightarrow \sup _{0 \leq x \leq c}\left|W^{0}(x)\right|, \quad \text { as } n \longrightarrow \infty \text {, }
$$

where $W^{0}$ is a standard Brownian bridge and $c:=v(T) /(1+v(T))$.

Proof. From (3.3), Theorems 3.2 and 3.3, it follows that

$$
\frac{\sqrt{c_{n}}}{1+\hat{v}_{n}} \frac{\hat{\mathscr{S}}_{n}-\mathscr{S}_{n}}{\hat{\mathscr{S}}_{n}}=\frac{M_{n}^{\varphi}}{\left(1+\hat{v}_{n}\right)} \cdot \frac{\mathscr{S}_{n}}{\hat{\mathscr{S}}_{n}} \Rightarrow \frac{W(v)}{1+v}, \quad \text { as } n \rightarrow \infty
$$

where $W$ denotes standard Brownian motion on $[0, T]$. So it is enough to see that $W(v) /$ $(1+v)$ and $W^{0}(v /(1+v))$ have the same distribution.

From the previous result, it follows that, for any $y>0$,

$$
\mathbb{P}\left[\sup _{0 \leq t \leq T}\left|\frac{\sqrt{c_{n}}}{1+\hat{v}_{n}(t)} \frac{\hat{\mathscr{S}}_{n}(t)-\mathscr{S}_{n}(t)}{\hat{\mathscr{S}}_{n}(t)}\right| \leq y\right] \rightarrow \mathbb{P}\left[\sup _{0 \leq x \leq c}\left|W^{0}(x)\right| \leq y\right], \quad \text { as } n \longrightarrow \infty .
$$

Then, the asymptotic $100(1-\alpha) \%$ confidence band for $\mathscr{S}_{n}$ in $[0, T]$ is

$$
\left[\hat{\mathscr{S}}_{n}(t)\left(1-\frac{1+\hat{v}_{n}(t)}{\sqrt{c_{n}}} e_{\alpha / 2}(c)\right), \hat{\mathscr{Y}}_{n}(t)\left(1+\frac{1+\hat{v}_{n}(t)}{\sqrt{c_{n}}} e_{\alpha / 2}(c)\right)\right], \quad 0 \leq t \leq T
$$

where $e_{\alpha / 2}(c)$ denotes the upper $(\alpha / 2)$-quantile of the distribution of $\sup _{0 \leq x \leq c}\left|W^{0}(x)\right|$.

Note that because of Theorem 2.1 and (2.15), the confidence band in (4.4) is also an asymptotic $100(1-\alpha) \%$ confidence band for $\omega_{n}$.

We have simulated the estimator process $\hat{\mathscr{S}}_{n}$ and the process $\mathscr{Y}_{n}$ in the time interval $[0,5]$. In the simulations we have assumed that the variables $U_{i}$ 's are uniformly distributed on the interval $(1,5)$ and that the $A_{n i}$ 's are uniformly distributed on the interval $(0.5,3.5)$. In order to construct the confidence band, we have estimated $c$ with

$$
\hat{c}:=\frac{\widehat{v}_{n}(T)}{1+\hat{v}_{n}(T)},
$$




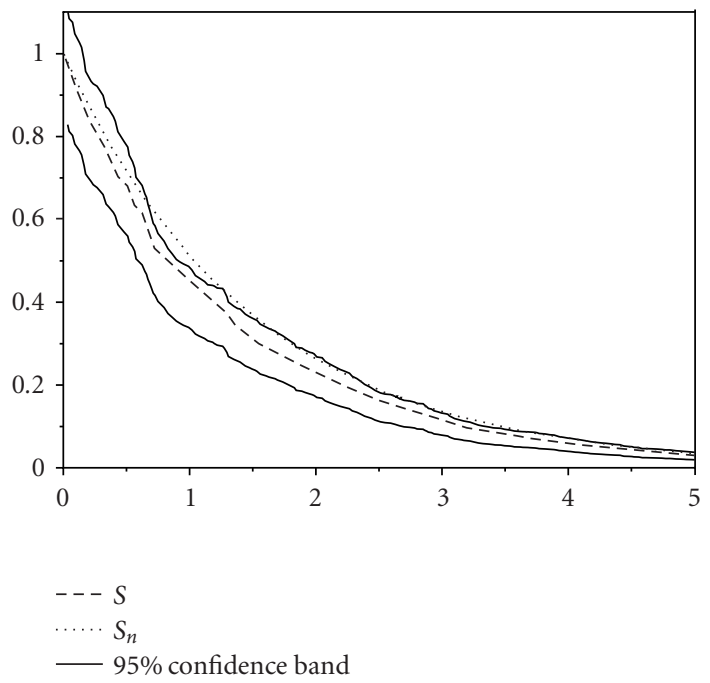

Figure 4.1. Comparison between $\mathscr{S}_{n}$ (dotted line), $\mathscr{S}$ (dashed line), and the $95 \%$ confidence band (continuous lines) for $c_{n}=100$.

and taken the value $e_{0.025}(\hat{c})$ from [10, Table 9]. The obtained results are illustrated in Figures 4.1 and 5.1 for $c_{n}=100$ and $c_{n}=1000$, respectively.

\section{The Poisson case}

In this section, we briefly discuss the case in which the dropping process $N_{n}$ is a homogeneous Poisson process with intensity measure $m_{n}$ given by

$$
m_{n}((s, t])=c_{n} \cdot \frac{t-s}{\mu_{U}}, \quad 0 \leq s<t
$$

In this case, the EFFAF does not depend on $n$ and is equal to $\mathscr{S}$ :

$$
\omega_{n}(t)=\mathscr{S}(t)=e^{-\left(\mu_{A} / \mu_{U}\right) t}, \quad t \geq 0 .
$$

So the problem reduces to an estimation problem for $\mathscr{Y}$. The chosen estimator $\hat{\mathscr{Y}}_{n}(t)$ is still defined as in (2.19). The following asymptotic results hold.

Theorem 5.1 (uniform consistency).

$$
\sup _{0 \leq t \leq T}\left|\hat{\mathscr{S}}_{n}(t)-\mathscr{S}(t)\right| \Longrightarrow 0, \quad \text { as } n \longrightarrow \infty
$$




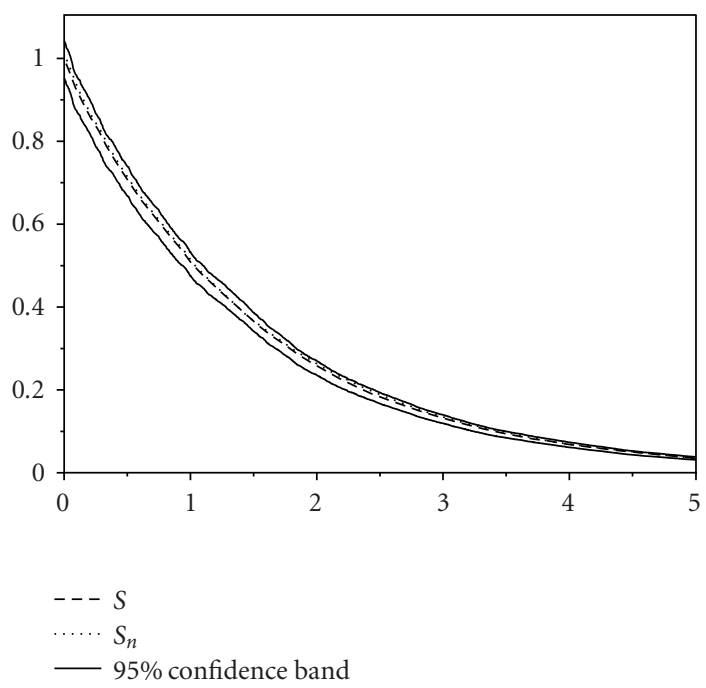

Figure 5.1. Comparison between $\mathscr{Y}_{n}$ (dotted line), $\mathscr{Y}$ (dashed line), and the $95 \%$ confidence band (continuous lines) for $c_{n}=1000$.

Theorem 5.2 (asymptotic Gaussianity). The process $\mathcal{M}_{n}^{\varphi}=\left\{\mathcal{M}_{n}^{\mathscr{P}}(t): 0 \leq t \leq T\right\}$, defined by

$$
\mu_{n}^{\mathscr{P}}(t):=\sqrt{{c_{n}}}\left(\frac{\hat{\mathscr{S}}_{n}(t)-\mathscr{Y}(t)}{\mathscr{S}(t)}\right), \quad 0 \leq t \leq T,
$$

converges to $W(v)$

$$
M_{n}^{\mathscr{S}} \Longrightarrow W(v), \quad \text { as } n \longrightarrow \infty,
$$

where $W$ is a standard Brownian motion on $[0, T]$, and $v=\left\{v(t):=\mu_{A}^{(2)} \cdot t / \mu_{U}, 0 \leq t \leq T\right\}$.

The variance function $v$ may be estimated by the uniform consistent estimator $\hat{v}_{n}$ defined by

$$
\widehat{v}_{n}(t):=\frac{1}{c_{n}} \sum_{i=1}^{N_{n}(t)} A_{n i}^{2}, \quad 0 \leq t \leq T .
$$

The band defined as in (4.4) is the asymptotic $100(1-\alpha) \%$ confidence band for $\mathscr{S}$ in $[0, T]$.

In the paper [6], the nonhomogeneous Poisson case is considered, that is,

$$
m_{n}((s, t])=c_{n} \cdot \int_{s}^{t} \lambda(u) d u, \quad 0 \leq s<t
$$


All results are obtained by extensive use of martingale theory. Furthermore, in the same paper, the estimation problem for the $\alpha(t)=\mu(t) \cdot \lambda(t)$ is solved by kernel smoothing method.

\section{References}

[1] P. K. Andersen, O. Borgan, R. D. Gill, and N. Keiding, Statistical Models Based on Counting Processes, Springer, New York, 1997.

[2] P. Billingsley, Convergence of Probability Measures, 2nd ed., Wiley Series in Probability and Statistics: Probability and Statistics, John Wiley \& Sons, New York, 1999.

[3] V. Capasso, M. De Giosa, and R. M. Mininni, Asymptotic properties of the maximum likelihood estimators of parameters of a spatial counting process modelling crystallization of polymers, Stochastic Analysis and Applications 13 (1995), no. 3, 279-294.

[4] V. Capasso, A. Micheletti, M. De Giosa, and R. M. Mininni, Stochastic modelling and statistics of polymer crystallization processes, Surveys on Mathematics for Industry 6 (1996), no. 2, 109-132.

[5] D. J. Daley and D. Vere-Jones, An Introduction to the Theory of Point Processes, Springer Series in Statistics, Springer, New York, 1988.

[6] M. De Giosa and R. M. Mininni, Free area estimation in a partially observed dynamic germ-grain model, Journal of Applied Mathematics and Stochastic Analysis 15 (2002), no. 4, 301-321.

[7] R. D. Gill and S. Johansen, A survey of product-integration with a view toward application in survival analysis, The Annals of Statistics 18 (1990), no. 4, 1501-1555.

[8] S. Karlin and H. M. Taylor, A First Course in Stochastic Processes, 2nd ed., Academic Press, New York, 1975.

[9] R. D. Ripley, Spatial Statistics, Wiley Series in Probability and Mathematical Statistics, John Wiley \& Sons, New York, 1981.

[10] M. Schumacher, Two-sample tests of Cramér-von Mises- and Kolmogorov-Smirnov-type for randomly censored data, International Statistical Review. Revue International de Statistique $\mathbf{5 2}$ (1984), no. 3, 263-281.

[11] D. Stoyan, W. S. Kendall, and J. Mecke, Stochastic Geometry and Its Applications, 2nd ed., John Wiley \& Sons, New York, 1995.

Marcello De Giosa: Dipartimento di Matematica, Università di Bari, Via Orabona 4, 70125 Bari, Italy E-mail address: mdegiosa@dm.uniba.it 


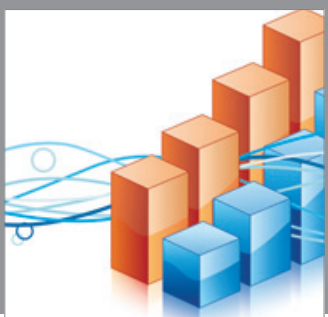

Advances in

Operations Research

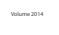

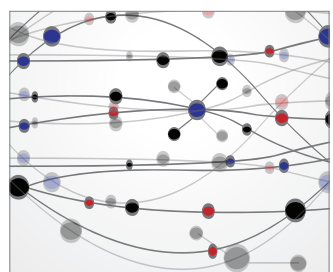

\section{The Scientific} World Journal
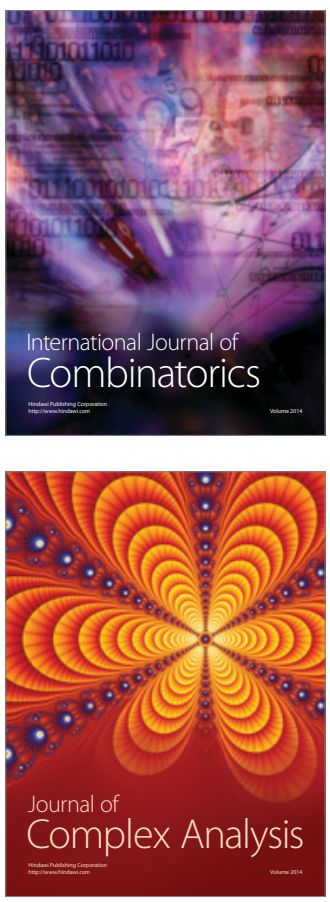

International Journal of

Mathematics and

Mathematical

Sciences
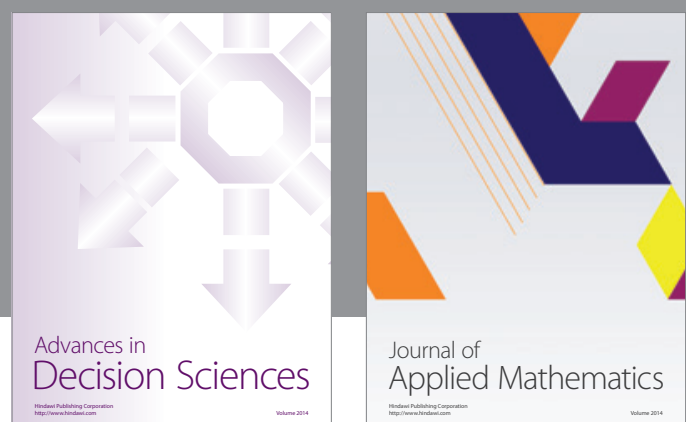

Journal of

Applied Mathematics
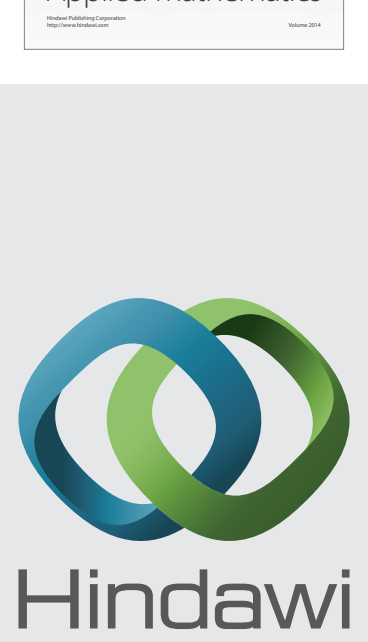

Submit your manuscripts at http://www.hindawi.com
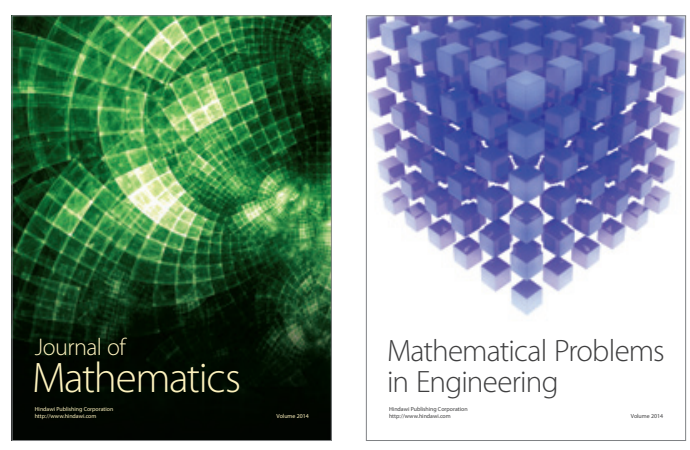

Mathematical Problems in Engineering
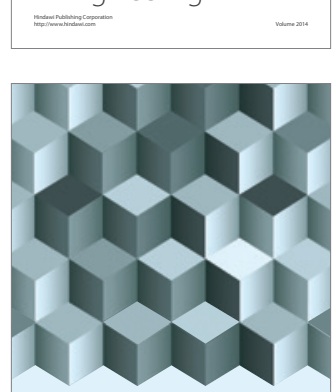

Journal of

Function Spaces
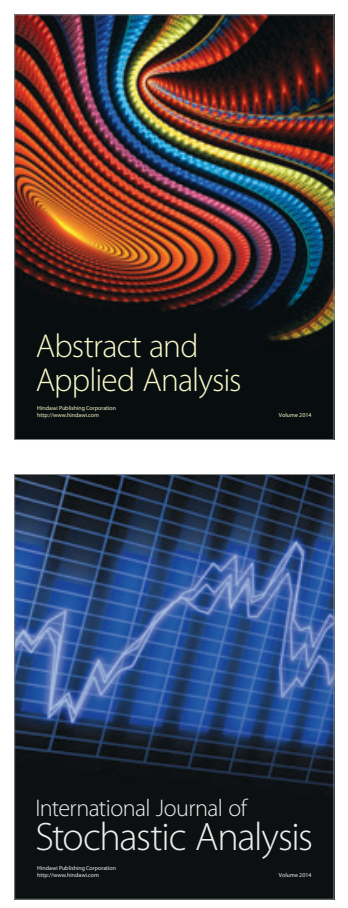

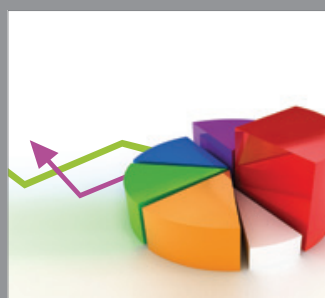

ournal of

Probability and Statistics

Promensencen
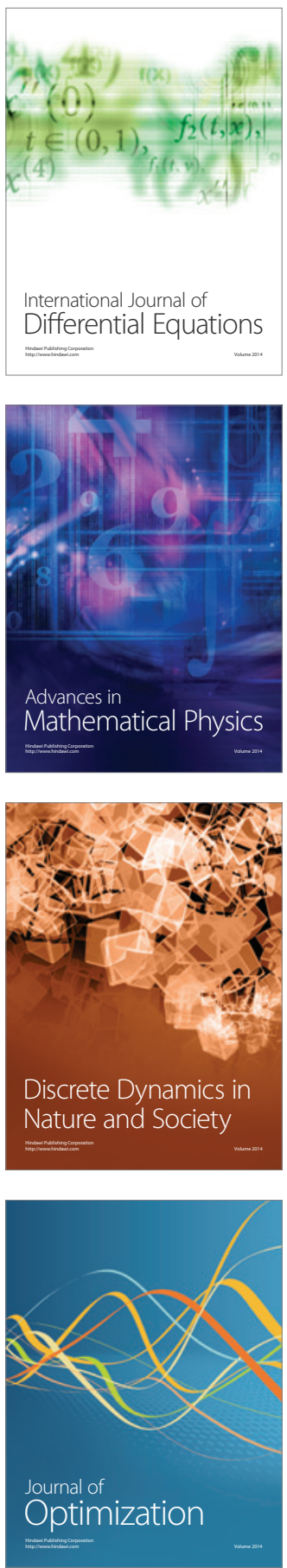